\title{
Genetic Risk Prediction for Normal-Karyotype Acute Myeloid Leukemia Using Whole-Exome Sequencing
}

\author{
Seong Gu Heo, Eun Pyo Hong, Ji Wan Park* \\ Department of Medical Genetics, Hallym University College of Medicine, Chuncheon 200-702, Korea
}

\begin{abstract}
Normal-karyotype acute myeloid leukemia (NK-AML) is a highly malignant and cytogenetically heterogeneous hematologic cancer. We searched for somatic mutations from 10 pairs of tumor and normal cells by using a highly efficient and reliable analysis workflow for whole-exome sequencing data and performed association tests between the NK-AML and somatic mutations. We identified 21 nonsynonymous single nucleotide variants (SNVs) located in a coding region of 18 genes. Among them, the SNVs of three leukemia-related genes (MUC4, CNTNAP2, and GNAS) reported in previous studies were replicated in this study. We conducted stepwise genetic risk score (GRS) models composed of the NK-AML susceptible variants and evaluated the prediction accuracy of each GRS model by computing the area under the receiver operating characteristic curve (AUC). The GRS model that was composed of five SNVs (rs75156964, rs56213454, rs6604516, rs10888338, and rs 2443878 ) showed $100 \%$ prediction accuracy, and the combined effect of the three reported genes was validated in the current study (AUC, $0.98 ; 95 \%$ confidence interval, 0.92 to 1.00 ). Further study with large sample sizes is warranted to validate the combined effect of these somatic point mutations, and the discovery of novel markers may provide an opportunity to develop novel diagnostic and therapeutic targets for NK-AML.
\end{abstract}

Keywords: acute myeloid leukemia, DNA sequence analysis, genetic variation, risk assessment

\section{Introduction}

Acute myeloid leukemia (AML), which is characterized by the rapid growth of abnormal myeloid cells in the marrow, is a highly malignant and cytogenetically heterogeneous type of cancer [1]. AML is categorized into three risk groups: favorable, intermediate, and unfavorable. AML with a normal karyotype (NK-AML) accounts for approximately $40 \%$ to $50 \%$ of adult patients and $25 \%$ of pediatric patients with AML and is composed of a heterogeneous group with an intermediate prognosis [2].

In the past few years, next-generation sequencing technologies have been developed rapidly in the field of cancer genomics $[3,4]$ and enabled a direct genome-wide association test for NK-AML at single-base resolution in an unbiased manner [5]. A comprehensive understanding of the genetic lesions is an important basis for developing personalized therapies for the treatment of NK-AML [6].

Ley et al. [5] carried out the first whole-genome association study for AML by using single-end whole- genome sequencing data and found 181 single nucleotide variations (SNVs), including 28 indels, nonsynonymous, and splicing sites (NPM1 and FLT3, respectively). Mardis et al. [7] performed paired-end whole-genome sequencing for cytogenetically normal AML, found seven nonsynonymous SNVs (nsSNVs), one splice site SNV, two indels in coding regions, and 52 somatic point mutations in conserved or regulatory genomic regions, and observed a $10 \%$ recurrence rate in the IDH1 mutation in 188 additional AML samples. In the following year, Ley et al. [5] carried out a case-control study of relapsed AML among patients who were reported in 2008. They found a one-base-pair deletion in the DNMT3A gene, which showed a recurrence risk of $22 \%$ in AML [8]. De Weer et al. [9] detected deletions within a chromosomal region of 7q35-q36, which contains the CNTNAP2 gene, in both Kasumi-3 and MUTZ-3 cell lines using high-resolution array comparative genomic hybridization (CGH). Particularly, Bejar et al. [10] identified novel associations between somatic mutations in two genes, ETV6 and GNAS, and both myelodysplastic syndrome (previously known as preleu- 
kemia) and AML using high-throughput genotyping technology. In the same year, Link et al. [11] identified a novel TP53 susceptibility mutation in AML patients through the use of whole-genome sequencing. Recent exome-sequencing studies found more than 60 susceptible variants, including indels and splicing site mutations, in AML patients [12]. However, to the best of our knowledge, none of the previous studies attempted to predict the risk of NK-AML by analyzing whole-exome sequencing data.

This study aimed to evaluate the effect of nsSNVs in 10 pairs of tumor and normal cells of NK-AML patients with whole-exome sequencing data. Finally, we developed genetic risk prediction models for NK-AML by creating genetic risk scores (GRS).

\section{Methods}

\section{Whole-exome sequencing data analysis}

A total of 20 DNA samples were obtained from 10 pairs of bone marrow (tumor) and germline (normal) cells collected from 10 NK-AML patients. We illustrated the workflow for whole-exome sequencing data analysis in Fig. 1. The raw

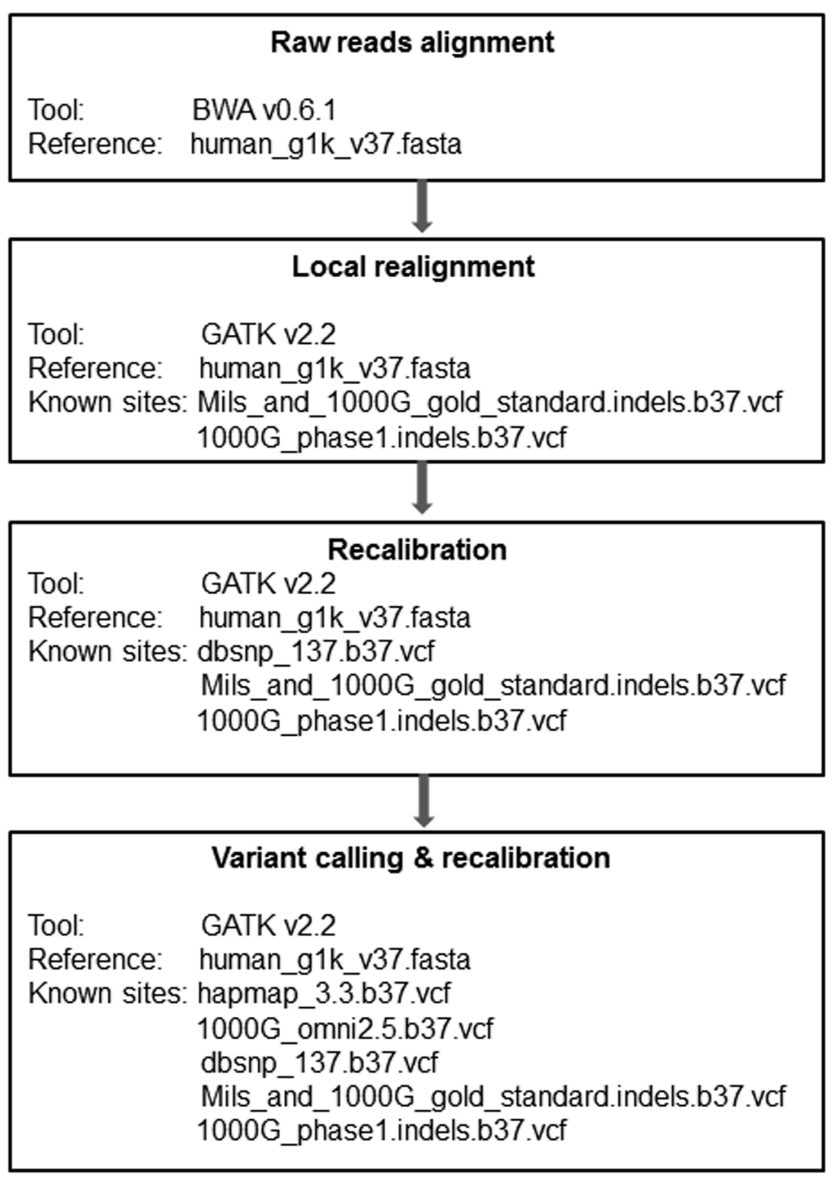

Fig. 1. Next-generation sequencing data analysis workflow. reads in FASTQ file format were mapped to the human reference, which was developed by the 1,000 genome project (human_g1k_v 37.fasta), by using the Burrows-Wheeler aligner (BWA v 0.6.1), which generates SAM format files [13]. The SAM files were converted into binary format files (BAM) by samtools v0.2.6, which reduces the file size and improves computing efficiency [14]. The read group information of the sequencing machine was added to the head of the BAM files. The aligned sequences were sorted in the order of chromosomes by Picard v1.79 (http:// picard.sourceforge.net) and went through a PCR duplicate marking process, which enables the Genome Analysis Toolkit (GATK) to ignore duplicates in subsequent processing [15]. Finally, the BAM files were indexed by bamtools v2.2.0 [16]. We performed a local realignment prior to recalibration, which gives the most accurate quality scores for each sample. Local realignment with known indel sites (Mills_and_1000G_gold_standard.indels.b37.vcf, 1000G_ phase1.indels.b37.vcf) for each individual does not require multiple sample realignments, which demand extreme computational power. However, we carried out a local realignment with the matched tumor and normal samples together to prevent misalignment due to the differences between these two tissue types. Recalibration was performed with multiple known sites (dbSNV_137.b37, Mills_and_1000G_gold_standard.indels.b37.vcf, and 1000G_ phase1.indels.b37.vcf), which may increase recalibration accuracy. We reduced the BAM file size to about $1 / 100$ of the original file size by using the GATK tool, which saved variant calling time without losing any essential information. We used the UnifiedGenotyper of GATK for variant calling, followed by variant recalibration with known sites (hapmap 3.3.b37.vcf, 1000G_omni2.5.b37.vcf, dbsnp_137.b37.vcf, and Mills_and_1000G_gold_standard.indels.b37.vcf), and annotated by them using snpEff v2.0.57 [17].

\section{Statistical analysis}

We performed logistic regression analyses between the somatic mutations and NK-AML using PLINK/SEQ v0.08 (http://atgu.atgu.mgh.harvard.edu/plinkseq), which provides powerful utilities in variant call format (vcf) for analyzing whole- exome and -genome data. Further, we verified the odd ratios and p-values estimated from PLINK/SEQ using Stata, v11.2 (Stata Corp., College Station, TX, USA).

We selected the somatic nsSNVs with complete call rates and evaluated the GRS models composed of the variants associated with NK-AML. The GRS was calculated for each individual by accumulating the number of risk alleles $(0,1$, or 2) of the SNVs. We created stepwise GRS models, comprised of the selected SNVs, according to their significance level; if the significance level was equal between two 
or more SNVs, we selected the SNVs in the order of their chromosomal position. In addition, we evaluated a GRS model that consisted of gene variants reported in previous leukemia studies $[9,10,18,19]$. We compared the area under the receiver operating characteristic curve (AUC) of each GRS model using the "roctab" and "roccomp" commands in Stata.

\section{Results}

The improvement in concordance between the empirical and reported quality scores of SNVs is shown in Fig. 2A. A significant improvement in the accuracy of quality scores after recalibration is shown in Fig. 2B. The association test for NK-AML yielded 42 SNVs that had a p-value of less than
(A)

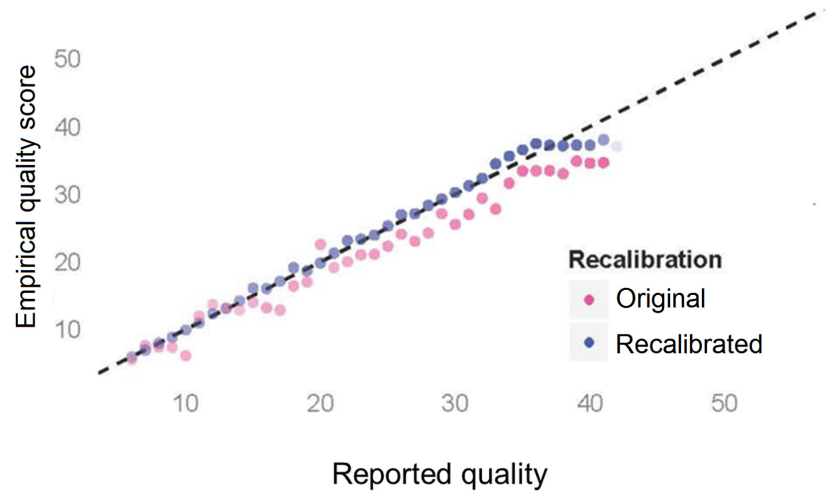

(B)

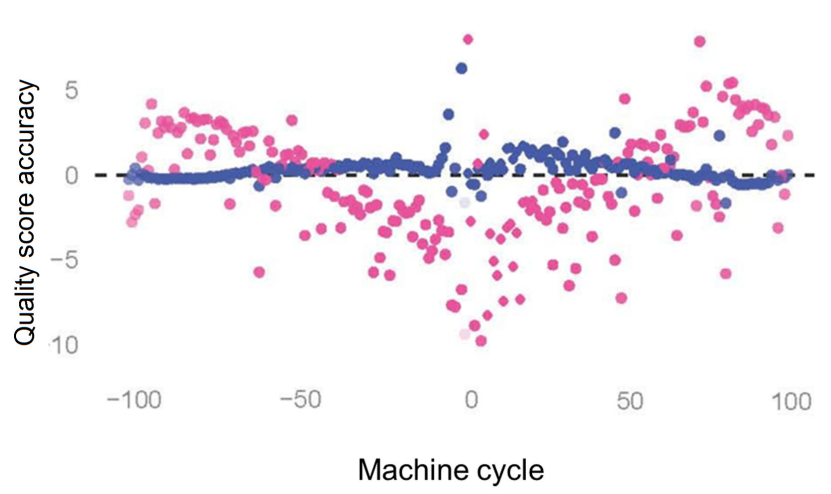

Fig. 2. Base quality score recalibration. Raw reads (pink) and recalibrated (blue) base quality scores for a patient. Panel (A) compares the reported base quality scores to the empirical estimates. Panel (B) shows the difference between the average reported and empirical quality scores for each machine cycle.

Table 1. Associations between $21 \mathrm{SNVs}$ and NK-AML in 10 pairs of tumor and normal samples

\begin{tabular}{|c|c|c|c|c|c|c|c|}
\hline Gene & Chr. & SNV & $\begin{array}{c}\text { Amino acid } \\
\text { change }\end{array}$ & $N / R$ & $\begin{array}{c}\text { RAF } \\
\text { case/control }\end{array}$ & OR $(95 \% \mathrm{Cl})$ & p-value \\
\hline PPIAL4G & $1 q 21.1$ & rs6604516 & Q24L & $\mathrm{A} / \mathrm{T}$ & $0.95 / 0.65$ & $21.0(1.8-248.1)$ & 0.016 \\
\hline LRRN2 & $1 q 32.1$ & rs201287849 & A141P & $\mathrm{C} / \mathrm{G}$ & $0.30 / 0.05$ & $13.5(1.2-152.2)$ & 0.035 \\
\hline OR2T33 & $1 q 44$ & rs10888338 & A169V & $\mathrm{A} / \mathrm{G}$ & $0.40 / 0.15$ & $9.3(1.2-73.0)$ & 0.033 \\
\hline \multirow[t]{2}{*}{ ANKRD36 } & $2 q 11.2$ & rs79579412 & K378R & $\mathrm{G} / \mathrm{A}$ & $0.95 / 0.70$ & $13.5(1.2-152.2)$ & 0.035 \\
\hline & & rs2443878 & K386E & $\mathrm{G} / \mathrm{A}$ & $0.85 / 0.60$ & $9.3(1.2-73.0)$ & 0.033 \\
\hline ATP13A3 & $3 q 29$ & rs75156964 & V55G & $\mathrm{C} / \mathrm{A}$ & $0.90 / 0.60$ & $16.0(1.8-143.2)$ & 0.013 \\
\hline \multirow[t]{2}{*}{ MUC4 } & $3 q 29$ & rs75459784 & $\mathrm{H} 2306 \mathrm{P}$ & $\mathrm{G} / \mathrm{T}$ & $0.85 / 0.60$ & $9.3(1.2-73.0)$ & 0.033 \\
\hline & & rs55824312 & P2192S & $\mathrm{A} / \mathrm{G}$ & $0.80 / 0.55$ & $13.5(1.2-152.2)$ & 0.035 \\
\hline TRIML1 & $4 q 35.2$ & rs200129069 & S254R & $\mathrm{C} / \mathrm{G}$ & $0.35 / 0.10$ & $9.3(1.2-73.0)$ & 0.033 \\
\hline CNTNAP2 & $7 q 35$ & rs200239604 & T589P & $\mathrm{C} / \mathrm{A}$ & $0.95 / 0.70$ & $13.5(1.2-152.2)$ & 0.035 \\
\hline PABPC1 & $8 q 22.2$ & rs79940439 & R475Q & $\mathrm{C} / \mathrm{T}$ & $0.40 / 0.15$ & $9.3(1.2-73.0)$ & 0.033 \\
\hline MUC5B & $11 \mathrm{p} 15.5$ & rs202160055 & R1097H & $\mathrm{G} / \mathrm{A}$ & $0.30 / 0.05$ & $13.5(1.2-152.2)$ & 0.035 \\
\hline TAS2R43 & $12 \mathrm{p} 13.2$ & rs201245949 & Q210H & $\mathrm{C} / \mathrm{G}$ & $0.40 / 0.15$ & $9.3(1.2-73.0)$ & 0.033 \\
\hline ATF7IP & $12 \mathrm{p} 13.1$ & rs199961592 & Q883K & $\mathrm{C} / \mathrm{A}$ & $0.45 / 0.20$ & $13.5(1.2-152.2)$ & 0.035 \\
\hline KIAA1033 & $12 q 24.11$ & rs199570381 & Q381K & $\mathrm{C} / \mathrm{A}$ & $0.30 / 0.05$ & $13.5(1.2-152.2)$ & 0.035 \\
\hline ATP8A2 & $13 q 12$ & rs201822155 & L624P & $\mathrm{G} / \mathrm{T}$ & $0.35 / 0.10$ & $9.3(1.2-73.0)$ & 0.033 \\
\hline L2HGDH & $14 q 21.3$ & rs201692645 & V198G & $\mathrm{A} / \mathrm{C}$ & $0.35 / 0.10$ & $9.3(1.2-73.0)$ & 0.033 \\
\hline LYPD5 & $19 q 13.31$ & rs79007092 & S151A & $\mathrm{A} / \mathrm{C}$ & $0.35 / 0.10$ & $9.3(1.2-73.0)$ & 0.033 \\
\hline \multirow[t]{2}{*}{ GNAS } & $20 q 13.3$ & rs56213454 & Q397P & $\mathrm{C} / \mathrm{A}$ & $0.90 / 0.60$ & $16.0(1.8-143.2)$ & 0.013 \\
\hline & & rs56371919 & M404I & $\mathrm{T} / \mathrm{G}$ & $0.90 / 0.65$ & $9.3(1.2-73.0)$ & 0.033 \\
\hline SUSD2 & $22 q 11$ & rs399140 & A91T & $\mathrm{A} / \mathrm{G}$ & $0.90 / 0.65$ & $9.3(1.2-73.0)$ & 0.033 \\
\hline
\end{tabular}

SNV, single nucleotide variation; NK-AML, normal-karyotype acute myeloid leukemia; Chr., chromosome; N/R, nonrisk/risk allele; $\mathrm{RAF}$, risk allele frequency; OR, odds ratio; $\mathrm{Cl}$, confidence interval.

${ }^{a}$ The rs75459784 is in complete linkage disequilibrium with the rs74420943. 
0.05 and passed the quality threshold of PLINK/SEQ (data not shown). Among 42 SNVs, we excluded 11 SNVs with no rs ID, four SNVs with call rates less than 1, and five synonymous SNVs for subsequent analysis.

A total of $21 \mathrm{nsSNVs}$ located in 18 candidate genes were selected and included in the GRS models after considering pairwise linkage disequilibrium (Table 1). This study replicated three somatic mutations of the MUC4, CNTNAP2, and GNAS genes, which were reported in previous studies on leukemia [9, 10, 18, 19]. In addition, we identified novel point mutations in 15 other genes. The SNVs rs75156964 (ATP13A3; odds ratio [OR], 9.33; $\mathrm{p}=0.013$ ) and rs56213454

Table 2. The area under the ROC curves for genetic risk score models consisting of SNVs

\begin{tabular}{ccll}
\hline Model $^{\mathbf{a}}$ & $\begin{array}{l}\text { No. of } \\
\text { SNVs }\end{array}$ & \multicolumn{1}{c}{ SNV } & AUC $(\mathbf{9 5 \% ~ C l})^{\mathbf{b}}$ \\
\hline 1 & 1 & rs75156964 & $0.80(0.62-0.98)$ \\
2 & 2 & Model1 + rs56213454 & $0.86(0.67-1.00)$ \\
3 & 3 & Model2 + rs6604516 & $0.92(0.81-1.00)$ \\
4 & 4 & Model3 + rs10888338 & $0.97(0.92-1.00)$ \\
5 & 5 & Model4 + rs2443878 & $1.00(1.00-1.00)$ \\
\hline
\end{tabular}

Note: SNVs were included in the model according to 1) the p-values and, 2) in case of the same significance levels, the chromosome in which the SNVs were located.

ROC, receiver operating characteristic; SNV, single nucleotide variation; $\mathrm{AUC}$, area under the ROC curve; $\mathrm{Cl}$, confidence interval.

${ }^{a}$ Genetic risk score model sums the numbers of risk alleles for each SNV $(0,1$, and 2$) ;{ }^{b} A \cup C s$ and $95 \%$ Cls were estimated from ROC analyses.
(GNAS; OR, 9.33; $\mathrm{p}=0.013$ ) showed the most significant evidence for association, and rs6604516 (PPIAL4G; OR, 21.00; $\mathrm{p}=0.016$ ) showed the strongest effect size among the 21 SNVs. Other SNVs also showed strong effects in the risk of NK-AML (OR, 9.33 to 13.5).

We compared the prediction accuracy of 21 stepwise GRS models. The predictive power for NK-AML reached $100 \%$ in model 5, which consisted of five nsSNVs: rs75156964 (ATP13A3, 3q29), rs56213454 (GNAS, 20q13.3), rs6604516 (PPIAL4G, 1q21.1), rs10888338 (OR2T33, 1q44), and rs2443878 (ANKRD36, 2q11.2) (Table 2, Fig. 3A). The five SNVs (rs75459784, rs55824312, rs200239604, rs56213454, and rs56371919) in three previously reported genesMUC4, CNTNAP2, and GNAS - also showed a high AUC value (AUC, 0.98; 95\% confidence interval [CI], 0.92 to 1.00).

\section{Discussion}

We evaluated the effects of 21 nsSNVs of 18 candidate genes for NK-AML in this study. Among 18 genes, we replicated previously reported associations of two genes (CNTNAP2 and GNAS) with AML $[9,10]$. The mutation in the MUC4 gene has been reported to be associated with acute lymphoblastic leukemia [18, 19]. The MUC4 gene encodes a mucin protein and a high-molecular-weight glycoprotein in humans. This integral membrane glycoprotein, which is observed on the cell surface, plays various roles in tumor progression [20]. Particularly, MUC4, complexed with
(A)

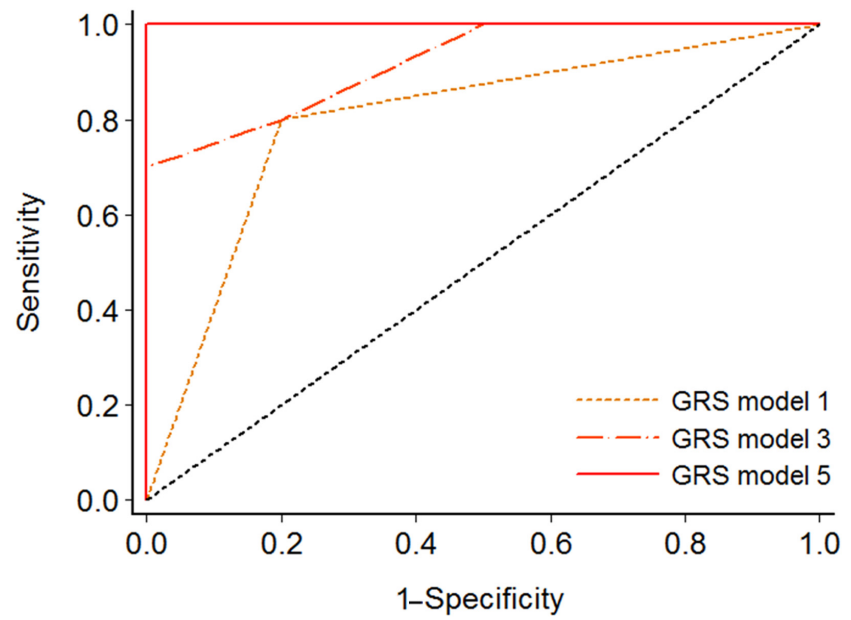

(B)

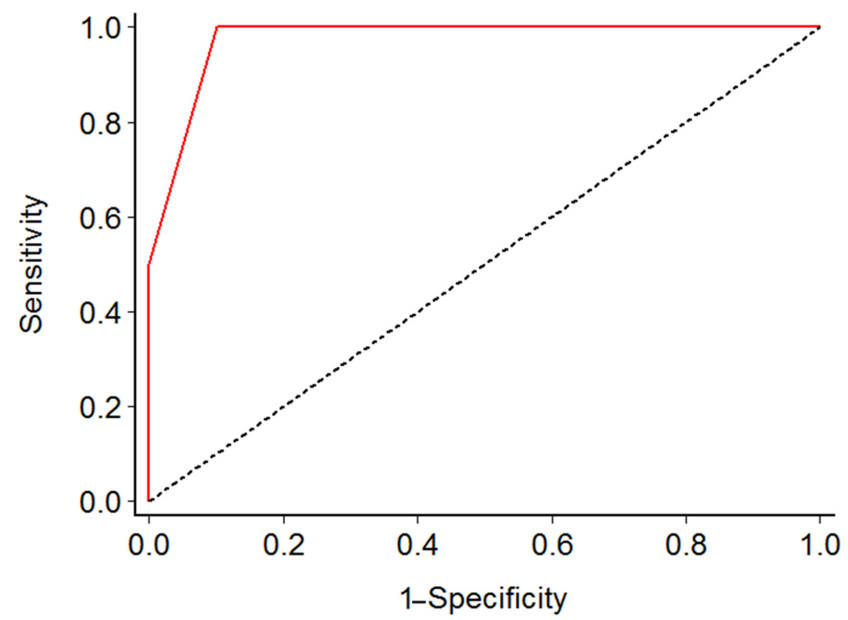

Fig. 3. Receiver operating characteristic analysis to measure the discriminatory power of each genetic risk score model in predicting normal-karyotype acute myeloid leukemia. (A) Model 1 (rs75156964), model 3 (rs75156964, rs56213454, and rs6604516), and model 5 (rs75156964, rs56213454, rs6604516, rs10888338, and rs2443878). (B) A model comprising five candidate single nucleotide variations (rs75459784, rs55824312, rs200239604, rs56213454, and rs56371919) in MUC4, CNTNAP2, and GNAS, reported in previous studies (area under the receiver operating characteristic curve, $0.98 ; 95 \%$ confidence interval, 0.92 to 1.00 ). GRS, genetic risk score. 
v-erb-b2 erythroblastic leukemia viral oncogene homolog 2 (ERBB2), results in the repression of apoptosis and the stimulation of proliferation; in addition, the overexpression of MUC4 in carcinoma cells promotes loss of cellular polarity by ERBB2-mediated disruption [21]. The CNTNAP2 gene is one of the largest genes in the entire human genome and occupies approximately $1.5 \%$ of chromosome 7 . This gene encodes a neurexin family member that functions in the vertebrate nervous system. The GNAS gene has a highly complex imprinted expression pattern. This gene provides instructions for making one component, the stimulatory alpha subunit, of a protein complex called a guanine nucleotide-binding protein (G-protein). G-protein alpha subunit regulates the cyclic AMP (cAMP) pathway. GNAS gene mutations are known to be associated with high cAMP signaling [22]. The novel associations between 15 somatic mutations (PPIAL4G, LRRN2, OR2T33, ANKRD36, ATP13A3, TRIML1, PABPC1, MUC5B, TAS2R43, ATF7IP, KIAA1033, ATP8A2 , L2HGDH, LYPD5, and SUSD2) and NK-AML need to be replicated, and their functional mechanisms in the AML should be investigated in future studies.

The GRS models that were comprised of the somatic nsSNVs showed extremely high predictive accuracy for the risk of NK-AML ( $86 \%$ to $100 \%$ ). The combination of five nsSNVs of previously reported genes (MUC4, CNTNAP2, and GNAS) had a predictive ability of $98 \%$ for the risk of NK-AML in this study. One of the limitations in this study was the small number of study subjects $(n=10)$, which resulted in substantial variations in the $95 \%$ CIs of the effects (ORs) of each SNV.

In conclusion, we have highlighted 21 susceptibility nsSNVs located in 18 genes. The GRS model that comprises five candidate SNVs is highly informative in predicting the risk for NK-AML. The discovery of novel markers may provide an opportunity to develop novel diagnostic and therapeutic targets for NK-AML. Further study with a larger sample size is necessary to validate the AML-related gene mutations and will provide an opportunity to develop a powerful genetic risk prediction model for NK-AML.

\section{Acknowledgments}

The sequence data for this study were provided by the National Project for Personalized Genomic Medicine (A111218-GM07) (PGM21 Project, A111218), Ministry for Health and Welfare, Republic of Korea.

\section{References}

1. Lowenberg B, Downing JR, Burnett A. Acute myeloid leukemia. N Engl J Med 1999;341:1051-1062.
2. Mrozek K, Marcucci G, Paschka P, Whitman SP, Bloomfield $\mathrm{CD}$. Clinical relevance of mutations and gene-expression changes in adult acute myeloid leukemia with normal cytogenetics: are we ready for a prognostically prioritized molecular classification? Blood 2007;109:431-448.

3. Stratton MR, Campbell PJ, Futreal PA. The cancer genome. Nature 2009;458:719-724.

4. Kwon SM, Cho H, Choi JH, Jee BA, Jo Y, Woo HG. Perspectives of integrative cancer genomics in next generation sequencing era. Genomics Inform 2012;10:69-73.

5. Ley TJ, Mardis ER, Ding L, Fulton B, McLellan MD, Chen K, et al. DNA sequencing of a cytogenetically normal acute myeloid leukaemia genome. Nature 2008;456:66-72.

6. Riva L, Luzi L, Pelicci PG. Genomics of acute myeloid leukemia: the next generation. Front Oncol 2012;2:40.

7. Mardis ER, Ding L, Dooling DJ, Larson DE, McLellan MD, Chen $\mathrm{K}$, et al. Recurring mutations found by sequencing an acute myeloid leukemia genome. N Engl J Med 2009;361: 1058-1066.

8. Ley TJ, Ding L, Walter MJ, McLellan MD, Lamprecht T, Larson DE, et al. DNMT3A mutations in acute myeloid leukemia. $N$ Engl J Med 2010;363:2424-2433.

9. De Weer A, Poppe B, Vergult S, Van Vlierberghe P, Petrick M, De Bock $\mathrm{R}$, et al. Identification of two critically deleted regions within chromosome segment 7q35-q36 in EVI1 deregulated myeloid leukemia cell lines. PLoS One 2010;5:e8676.

10. Bejar R, Stevenson K, Abdel-Wahab O, Galili N, Nilsson B, Garcia-Manero G, et al. Clinical effect of point mutations in myelodysplastic syndromes. N Engl J Med 2011;364:24962506.

11. Link DC, Schuettpelz LG, Shen D, Wang J, Walter MJ, Kulkarni $\mathrm{S}$, et al. Identification of a novel TP53 cancer susceptibility mutation through whole-genome sequencing of a patient with therapy-related AML. JAMA 2011;305:1568-1576.

12. Yan XJ, Xu J, Gu ZH, Pan CM, Lu G, Shen Y, et al. Exome sequencing identifies somatic mutations of DNA methyltransferase gene DNMT3A in acute monocytic leukemia. Nat Genet 2011;43:309-315.

13. Li H, Durbin R. Fast and accurate long-read alignment with Burrows-Wheeler transform. Bioinformatics 2010;26:589-595.

14. Li H, Handsaker B, Wysoker A, Fennell T, Ruan J, Homer N, et al. The Sequence Alignment/Map format and SAMtools. Bioinformatics 2009;25:2078-2079.

15. McKenna A, Hanna M, Banks E, Sivachenko A, Cibulskis K, Kernytsky A, et al. The Genome Analysis Toolkit: a MapReduce framework for analyzing next-generation DNA sequencing data. Genome Res 2010;20:1297-1303.

16. Barnett DW, Garrison EK, Quinlan AR, Stromberg MP, Marth GT. BamTools: a C++ API and toolkit for analyzing and managing BAM files. Bioinformatics 2011;27:1691-1692.

17. Cingolani P, Platts A, Wang le L, Coon M, Nguyen T, Wang L, et al. A program for annotating and predicting the effects of single nucleotide polymorphisms, SnpEff: SNPs in the genome of Drosophila melanogaster strain w1118; iso-2; iso-3. Fly (Austin) 2012;6:80-92.

18. Harvey RC, Mullighan CG, Wang X, Dobbin KK, Davidson GS, Bedrick EJ, et al. Identification of novel cluster groups in pe- 
diatric high-risk B-precursor acute lymphoblastic leukemia with gene expression profiling: correlation with genome-wide DNA copy number alterations, clinical characteristics, and outcome. Blood 2010;116:4874-4884.

19. Zhang J, Mullighan CG, Harvey RC, Wu G, Chen X, Edmonson $\mathrm{M}$, et al. Key pathways are frequently mutated in high-risk childhood acute lymphoblastic leukemia: a report from the Children's Oncology Group. Blood 2011;118:30803087.

20. Srivastava SK, Bhardwaj A, Singh S, Arora S, Wang B, Grizzle
WE, et al. MicroRNA-150 directly targets MUC4 and suppresses growth and malignant behavior of pancreatic cancer cells. Carcinogenesis 2011;32:1832-1839.

21. Kufe DW. Mucins in cancer: function, prognosis and therapy. Nat Rev Cancer 2009;9:874-885.

22. Horvath A, Boikos S, Giatzakis C, Robinson-White A, Groussin L, Griffin KJ, et al. A genome-wide scan identifies mutations in the gene encoding phosphodiesterase 11A4 (PDE11A) in individuals with adrenocortical hyperplasia. Nat Genet 2006;38:794-800. 